Известия НАН Армении, Физика, т.57, №1, с.131-140 (2022)

УДК 548.732

DOI:10.54503/0002-3035-2022-57.1-131

\title{
КРИВЫЕ КАЧАНИЯ РЕНТГЕНОВСКОЙ АСИММЕТРИЧНОЙ ЛАУЭ ДИФРАКЦИИ В КРИСТАЛЛЕ С КУБИЧЕСКИ- НЕЛИНЕЙНЫМ ОТКЛИКОМ
}

\author{
М.К. БАЛЯН* \\ Ереванский государственный университет, Ереван, Армения \\ *e-mail: mbalyan@ysu.am \\ (Поступила в редакцию 8 сентября 2021 г.)
}

\begin{abstract}
Рассмотрена рентгеновская асимметричная Лауэ дифракция в идеальном кристалле с плоской входной поверхностью с учетом кубически-нелинейного отклика кристалла. С использованием точных решений изучены кривые качания в зависимости от угла отклонения от точного направления Брэгга и от интенсивности падающей волны. Исследована зависимость кривых качаний от степени асимметричности геометрии дифракции. Полученные результаты, являясь одним из важных характеристик нелинейной динамической дифракции, могут быть использованы для получения интенсивных рентгеновских пучков с заданными параметрами, а так же для исследования объектов и кристаллических деформаций.
\end{abstract}

\section{1. Введение}

Коэффициенты прохождения и отражения динамически-дифрагированной рентгеновской плоской волны на выходной поверхности кристалла зависят от параметра отклонения от угла Брэгга, от толщины кристаллической пластинки и от степени асимметрии геометрии дифракции. Что касается случаю пучков высокой интенсивности, какими являются пучки от синхротронных источников рентгеновского излучения третьего поколения или же от рентгеновских лазеров на свободных электронах, то необходимо учесть нелинейный член третьего порядка в поляризуемости кристалла. Тогда одним из параметров задачи становится также интенсивность падающего пучка. Брэгговская дифракция используется как для изучения объектов, так и для получения пучков с заданными параметрами. Последнее значит приготовить пучки с требуемой степенью монохроматичности, коллимации, либо же сфокусировать пучок. Но в случае пучков с высокой интенсивностью необходимо исследование брэгговской нелинейной дифракции. Одним из важных особенностей брэгговской нелинейной дифракции являются кривые качания, т.е. при фиксированной толщине и угле асимметрии зависимость коэффициентов прохождения и отражения от отклонения от угла Брэгга $[1,2]$, а также, в нелинейном случае, от интенсивности падающей волны. В работе [3] получены основные уравнения динамической рентгеноской дифракции в кристалле с кубически-нелинейным откликом на внешнее электромагнитное поле. 
Для случая симметричного отражения по Брэггу точное решение найдено в работе [4]. Точное решение в случае Лауэ, учитывающее отклонение от угла Брэгга, а так же асимметричность дифракции, найдено в работе [5]. Эти найденные решения позволяли исследовать нелинейную дифракцию в кристалле и найти основные ее закономерности. Оказалось, что в нелинейном случае тоже имеет место маятниковый эффект. Для длины экстинкции было найдено аналитическое выражение. Уравнения динамической нелинейной дифракции можно решать также численно. В работе [6] численно была исследована нелинейная динамическая дифракция третьего порядка для падающего пучка с ограниченным фронтом волны. Исследование нелинейной дифракции третьего порядка рентгеновских импульсов было проведено в работе [7].

В настоящее время проводятся теоретические и экспериментальные исследования также других, как второго, так и третьего порядка нелинейных рентгеновских эффектов. Параметрическое преобразование с понижением частоты является нелинейным эффектом второго порядка. Сущность заключается в том, что первоначальный фотон, из-за нелинейного отклика кристалла, распадается на два фотона более низкой частоты, так что частота первоначального фотона равна сумме частот рожденных фотонов. В области рентгеновских частот этот эффект впервые был предсказан в работе [8]. Эффект экспериментально наблюдался и изучался в работах [9-19] с использованием как лабораторных, так и синхротронных источников рентгеновского излучения. Другой рентгеновский нелинейный эффект второго порядка - генерация второй гармоники, теоретически изучался в работе [20], а экспериментально наблюдался в работе [21]. Нелинейный эффект Комптона экспериментально изучался в работе [22], а теоретически - в работе [23]. Рентгеновская кубически-нелинейная одноволновая дифракция теоретически изучалась в работе [24], нелинейное смешивание четырех волн - в работе [25], рентгеновское двухфотонное поглощение - в работе [26].

В настоящей работе, на основе полученных точных решений в работе [5], изучаются кривые качания в случае кубически-нелинейной асимметричной дифракции по Лауэ в совершенном кристалле. Кривые качания исследуются как в зависимости от отклонения от угла Брэгга, так и в зависимости от интенсивности падающей волны.

\section{2. Основные формулы}

Схема асимметричной дифракции по Лауэ показана на рис.1. Отражающие плоскости RP отклонены от перпендикулярного к входной поверхности положении на угол асимметрии $\alpha$, который в случае дифракции на прохождение, меняется в пределах $|\alpha|<\pi / 2-\theta$. Этот угол считается положительным, если отклонение от перпендикулярного к входной поверхности положения происходит против вращения часовой стрелки, и отрицательным, при отклонении в противоположном направлении. Плоская рентгеновская б-поляризованная волна с амплитудой $E^{i}$ падает на кристалл под углом скольжения $\theta^{i}$ по отношению к отражающим плоскостям, близким к углу Брэгга. В кристалле возникают две 


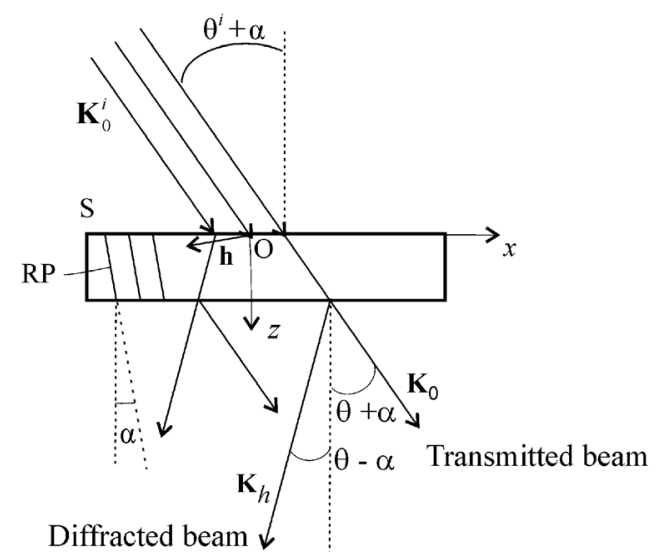

Рис.1. Схема асимметричной дифракции Лауэ: $\mathbf{K}_{0}^{i}-$ средний волновой вектор падающей волны, $\theta^{i}$ - угол между волновым вектором и отражающими плоскостями, $\theta-$ угол Брэгга, $\alpha-$ угол между отражающими плоскостями RP и внутренней нормалью к входной поверхности $\mathrm{S}$, $\mathrm{O} x z$ - координатная система в плоскости дифракции, ось Оy направлена перпендикулярно к плоскости дифракции по закону правой системы координат, $\mathbf{K}_{0}$ и $\mathbf{K}_{h}=\mathbf{K}_{0}+\mathbf{h}-$ волновые векторы проходящей и дифрагированной волн, удовлетворяющие точному условию Брэгга, $\mathbf{h}-$ вектор дифракции, перпендикулярный к отражающим плоскостям.

сильные волны - проходящая и дифрагированная, с амплитудами $E_{0}$ и $E_{h}$. Считается, что толщина кристалла достаточно мала и можно пренебречь поглощением, т.е. $\mu z<1$, где $\mu-$ коэффициент поглощения кристалла. Считается также, что интенсивность падающей волны может быть настолько высокой, что нужно учитывать кубически-нелинейный член в выражении поляризуемости кристалла. Кроме того, считается что отражение с вектором дифракции $2 \mathbf{h}$ запрещено, т.е. соответствующие коэффициенты Фурье как линейной, так и нелинейной поляризуемости равны нулю.

Как было сказано во введении, в работе [5] найдены точные решения. Будем исследовать коэффициенты прохождения $T(z)=\left|E_{0}\right|^{2} /\left|E^{i}\right|^{2}$ и отражения $R(z)=\gamma_{h}\left|E_{h}\right|^{2} /\left(\gamma_{0}\left|E^{i}\right|^{2}\right)$, основываясь на эти точные решения. Коэффициенты прохождения и отражения зависят от знака $Q$ (определение $Q$ - чуть ниже). Необходимо различать следующие случаи: $Q>0, Q<0, Q=0$ и $q_{1}<0, Q=0$ и $q_{1}>0$. Здесь приняты следующие обозначения [5]:

$$
Q=q_{1}^{2} / 4+q_{2}^{3} / 27,
$$

$q_{1}=2(a / 3)^{3}-a b_{1} / 3+c, \quad q_{2}=-a^{2} / 3+b_{1}, \quad a=\beta_{2} / \beta_{1}, \quad b_{1}=\beta_{3} / \beta_{1}, \quad b_{1}=\beta_{3} / \beta_{1} \quad$ и $c=\beta_{4} / \beta_{1} . \quad$ Причем $\quad \beta_{1}=\left(\alpha_{4}^{2}-\alpha_{2}\right), \quad \beta_{2}=I_{1}\left(\alpha_{2}-\alpha_{4}^{2}\right)+2 \alpha_{3} \alpha_{4}-\alpha_{1}$, $\beta_{3}=1+I_{1}\left(\alpha_{1}-2 \alpha_{3} \alpha_{4}\right)+\alpha_{3}^{2}$ и $\beta_{4}=-I_{1} \alpha_{3}^{2}$. Входящие здесь коэффициенты $\alpha_{i}$ определяются как $\alpha_{1}=(1-b)\left[2+I_{1}(1-b) /\left(1-I_{1}\right)\right] /\left(1-I_{1}\right), \quad \alpha_{2}=-(1-b)^{2} /\left(1-I_{1}\right)^{2}$, $\alpha_{3}=-b^{1 / 2} \sin 2 \theta\left[\Delta \theta-\chi_{0}(1-b)\left[1-I_{1}(1+b) / 2\right] /(2 b \sin 2 \theta)\right] /\left[\left|\chi_{h}\right|\left(1-I_{1}\right)\right], \quad \alpha_{4}=$ 
$\left|\chi_{0}\right|\left(1-4 b+b^{2}\right) /\left[4 b^{1 / 2}\left|\chi_{h}\right|\left(1-I_{1}\right)\right]$. В эти выражения входят $b=\gamma_{0} / \gamma_{h}-$ коэффициент асимметричности, $\gamma_{0, h}=\cos (\theta \pm \alpha)$ - направляющие косинусы волнового вектора падающей волны на направление внутренней нормали к входной поверхности кристалла, $\theta-$ угол Брэгга, $\chi_{0, h}-$ Фурье коэффициенты поляризуемости кристалла для нулевого вектора обратной решетки и для вектора отражения $\mathbf{h}$, $y=y_{0} /\left(1-I_{1}\right), y_{0}=b^{1 / 2} \sin 2 \theta\left[\Delta \theta-\chi_{0}(1-b) /(2 b \sin 2 \theta)\right] /\left|\chi_{h}\right|$ - параметр отклонения от условия Брэгга, $\Delta \theta=\theta^{i}-\theta$ - угол отклонения от угла Брэгга, $I_{1}=I / I_{\max }$ - нормированная критической интенсивностью $I_{\max }$ интенсивность падающей волны $I=\left|E_{0}^{i}\right|^{2}, E_{0}^{i}$ - постоянная амплитуда падающей волны, $I_{\max } \approx 2.1 \cdot 10^{25} \mathrm{~B}^{2} / \mathrm{M}^{2}$ — интенсивность, при которой соответствующая напряженность рентгеновской волны $E_{\max }=\sqrt{I_{\max }} \approx 4.6 \cdot 10^{12} \mathrm{~B} / \mathrm{M}$ равняется напряженности атомного ядра на расстоянии боровского радиуса (выражение для этой величины см. в [5] и в ссылках, данных там).

Коэффициенты прохождения и отражения зависят от угла отклонения от точного угла Брэгга, от интенсивности падающего пучка и от угла, составленного отражающими плоскостями и нормалью к входной поверхности кристалла. Ниже исследуются коэффициенты прохождения и отражения в зависимости от параметра отклонения и от интенсивности падающей волны при фиксированной толщине кристалла и для различных значениях угла асимметрии отражающих плоскостей.

\section{3. Кривые качания кубически-нелинейной асимметричной дифракции по Лауэ}

Кривые качания исследуем для отражения $\operatorname{Si}(111)$ с длиной волны излучения $\lambda=0.71 \AA$, падающая волна плоская и $\sigma$-поляризованная (вектор электрического поля перпендикулярна к плоскости дифракции). В этом случае отражение $\mathrm{Si}(222)$ запрещенное и можно применить приведенные выше формулы для таких толщин, для которых можно пренебрегать поглощением.

\section{1. Кривые качания в зависимости от углового отклонения от условия Брэгга}

Сначала рассмотрим случай $\alpha=-60^{\circ}(b=1.49)$. Для сравнения линейного и нелинейного случаев, на рис.2а, для интенсивности $I_{1}=10^{-5}$, что фактически соответствует линейному случаю, показаны кривые качания прохождения и отражения в зависимости от углового отклонения $\Delta \theta$ от точного угла Брэгга (на графике углы даются в угловых секундах, причем этому интервалу углов соответствует промежуток [-2,2] параметра $y_{0}$ ), причем $Q>0$. Выбрана толщина $z=2 \Lambda_{\mathrm{NL}} \approx 40.6$ мкм при параметре отклонения $y_{0}=0$. Здесь $\Lambda_{\mathrm{NL}}$ длина экстинкции нелинейного случая для $Q>0$ [5]. Эта величина, в отличие от линейного случая, существенно зависит и от интенсивности падающей волны. Так как интенсивность настолько мала, что реализуется линейный случай, то экстинкционная длина нелинейного случая совпадает с экстинкционной длиной линейного случая $[1,2]$ 

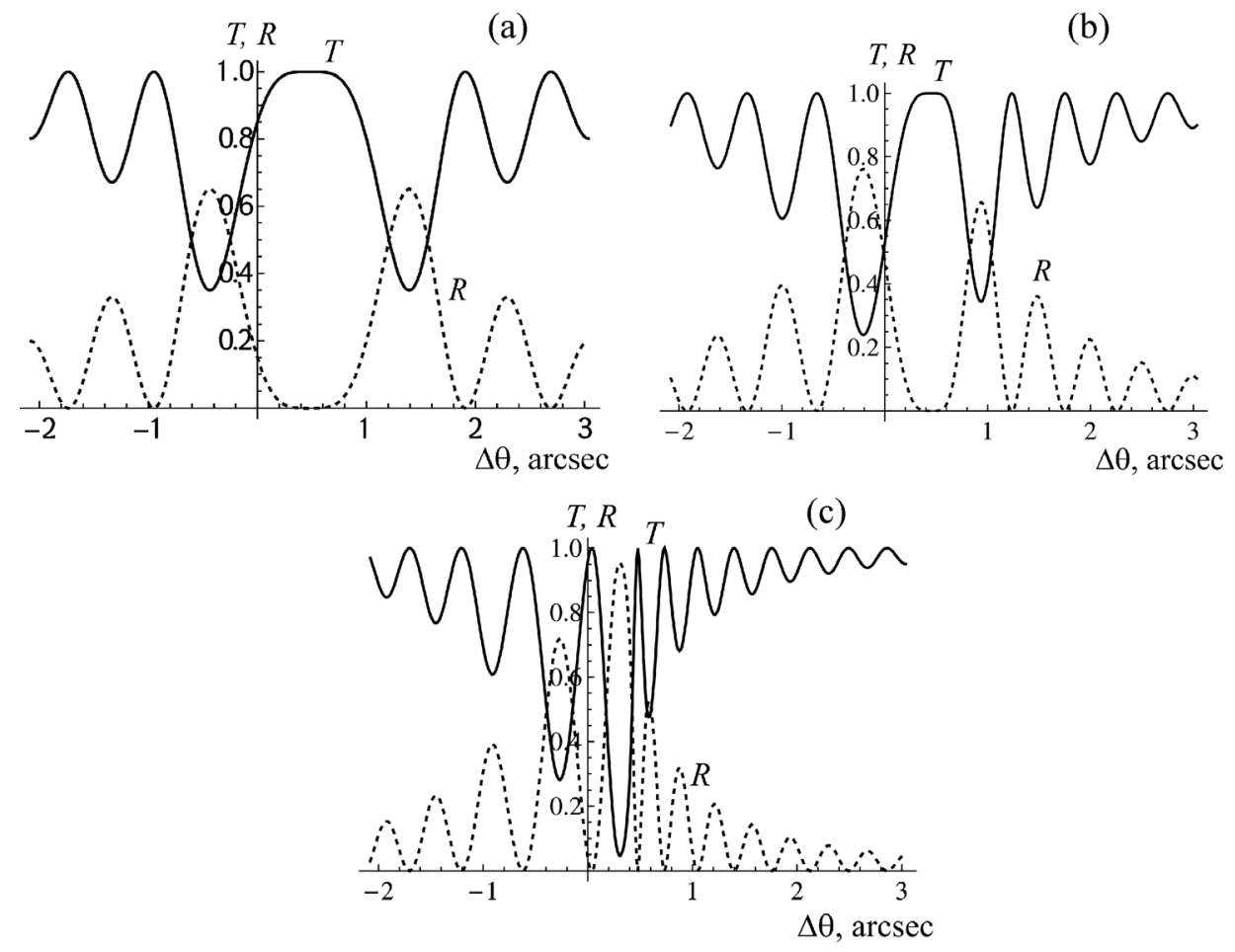

Рис.2. Кривые качания для случая $\alpha=-60^{\circ}$, сплошные кривые - коэффициент прохождения, пунктир - коэффициент отражения. (а) Линейный случай, (b) $I_{1}=0.2$, и (c) $I_{1}=0.4$.

$$
\Lambda_{\mathrm{L}}==\frac{\lambda\left(\gamma_{0} \gamma_{h}\right)^{1 / 2}}{\left|\chi_{h}\right| \sqrt{1+y_{0}^{2}}},
$$

которая зависит от параметра отклонения и не зависит от интенсивности. Отметим, что для выбранной толщины $\mu z=0.06$. Так как толщина выбрана равной двух экстинкционных длин, то значению $y_{0}=0$ соответствует максимум коэффициента прохождения и минимум коэффициента отражения, соответствующие угловому отклонению $\Delta \theta=\chi_{0}(1-b) /\left(2 b \sin 2 \theta\right.$ ) (в этой точке $\left.y_{0}=0\right)$. Это хорошо известно в линейной теории как коррекция преломления на выполнение условия Брэгга $[1,2]$. Посмотрим, как изменятся кривые качания при возрастании интенсивности. На рис. $2 \mathrm{~b}$ и $2 \mathrm{c}$ показаны кривые качания для интенсивностей $I_{1}=0.2 \quad$ и $I_{1}=0.4, \quad$ для глубин $\quad z=2 \Lambda_{\mathrm{NL}}\left(y_{0}=0, I_{1}=0.2\right) \approx 54.9$ мкм $\quad$ и $z=2 \Lambda_{\mathrm{NL}}\left(y_{0}=0, I_{1}=0.4\right) \approx 71.8$ мкм, соответственно. Отметим, что $\mu z=0.08$ и $\mu z=0.1$. Для этих значений параметров $Q>0$. Экстинкционные глубины вычислены по соответствующей формуле из [5]. Как видно из этих рисунков, ширины главных максимумов прохождения и главных минимумов отражения сужаются, а координаты их центров уменьшаются. Такое смещение координат объясняется тем, что как было показано в работе [5], роль параметра отклонения, с учетом интенсивности, играет 


$$
\alpha_{3}=-b^{1 / 2} \sin 2 \theta\left[\Delta \theta-\chi_{0}(1-b)\left[1-I_{1}(1+b) / 2\right] /(2 b \sin 2 \theta)\right] /\left[\left|\chi_{h}\right|\left(1-I_{1}\right)\right]
$$

(определение $\alpha_{3}$ см. объяснения после формулы (1)), так что главный максимум и минимум смещаются в точку $\Delta \theta=\chi_{0}(1-b)\left[1-I_{1}(1+b) / 2\right] /(2 b \sin 2 \theta)$, соответствующее значению $\alpha_{3}=0$. Ясно, что новому положению главных максимумов и минимумов уже не отвечает $y_{0}=0$. Из этих рисунков видно также появление асимметрии главных максимумов и минимумов, а также периодов осцилляций по обе стороны главных максимумов и минимумов.

Кривые качания в зависимости от параметра отклонения $y_{0}$, для случая $\alpha=60^{\circ}(b=0.67)$ приведены на рис.3. Зависимости даны от угла отклонения, причем показанному отрезку углов соответствует отрезок $[-2,2]$ параметра отклонения $y_{0}$. На рис.За показана кривая качания, соответствующая линейной теории: интенсивность падающей волны $I_{1}=10^{-5}$. Глубина взята равной $z=40.6$ мкм, что соответствует двум экстинкционным длинам линейного случая (2) для параметра отклонения $y_{0}=0$. Эта глубина в линейной теории не отличается от соответствующей величины случая $\alpha=-60^{\circ}$. Так как $b<1$, то из за преломления главный максимум проходящего пучка и главный минимум дифрагированного пучка смещены в противоположную сторону, по сравнению со случаем $b>1$, показанной на рис.2а. Этой точке соответствует параметр $y_{0}=0$ и кроме того, ширины главного максимума и минимума больше, по сравнению со случаем $\alpha=-60^{\circ}$. Это хорошо известные в линейной теории факты $[1,2]$. Теперь опять
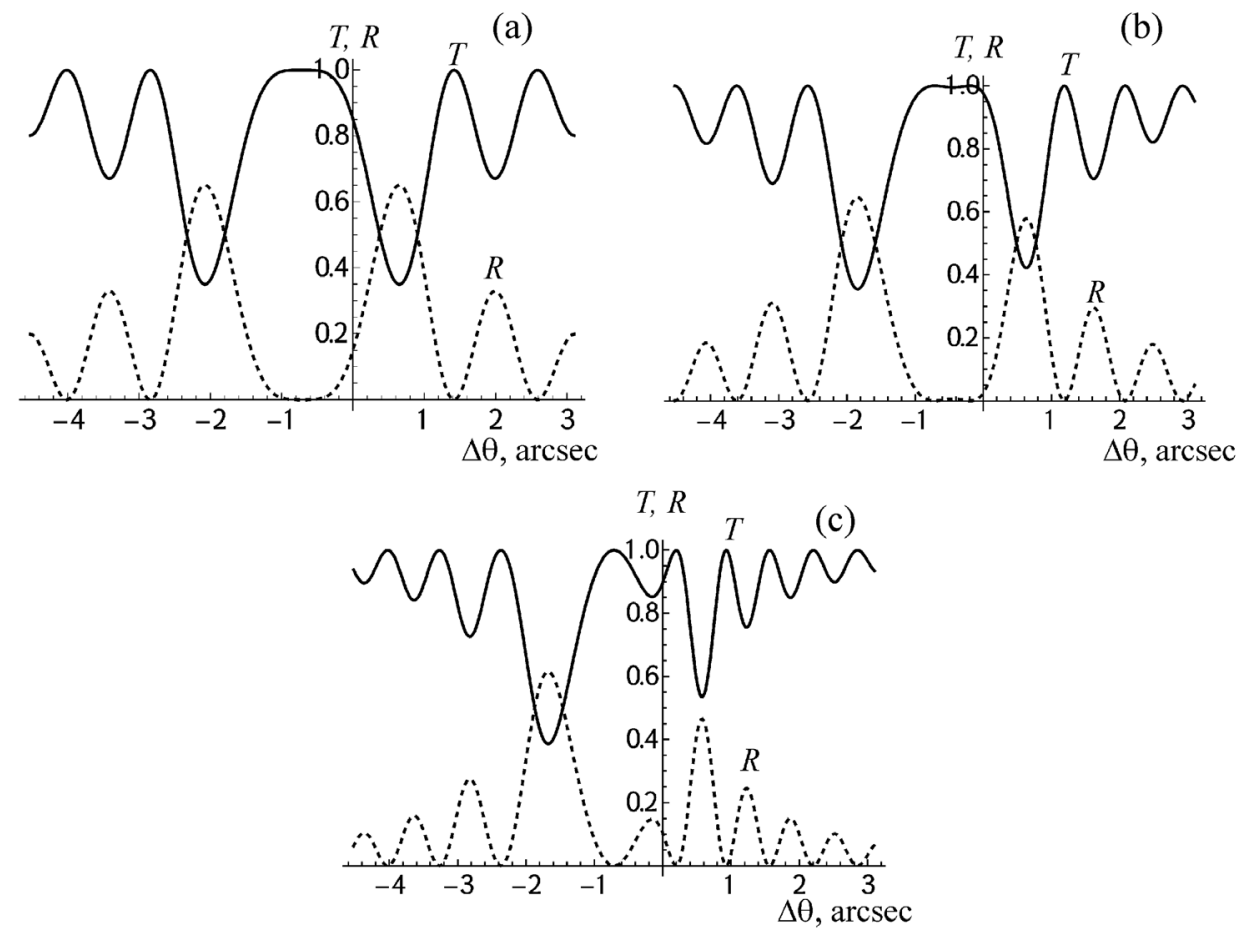

Рис.3. Кривые качания для случая $\alpha=60^{\circ}$, сплошные кривые - коэффициент прохождения, пунктир - коэффициент отражения. (а) Линейный случай, (b) $I_{1}=0.2$ и (c) $I_{1}=0.4$. 
следим за изменением кривых качаний увеличивая интенсивность падающей волны. На рис.3b и 3 с показаны кривые качания для значений интенсивности $I_{1}=0.2$ и $I_{1}=0.4$. Как видно из этих рисунков, опять же ширины главных максимумов и минимумов уменьшаются, а координаты главных пиков и главных минимумов увеличиваются, в противовес случая $b>1$. Это опять же объясняется тем, что в нелинейном случае координаты главных пиков и минимумов определяются из $\Delta \theta=\chi_{0}(1-b)\left[1-I_{1}(1+b) / 2\right] /(2 b \sin 2 \theta)$. В этих примерах $Q>0$. Глубины взяты 48.5 мкм и 59.6 мкм, которые соответствуют двум экстинкционным длинам нелинейного случая для значения $y_{0}=0$. На этих глубинах поглощение ничтожное и можно применять приведенные выше формулы для коэффициентов прохождения и отражения.

\section{2. Кривые качания в зависимости от интенсивности}

В отличие от линейного случая, в нелинейном случае коэффициенты прохождения и отражения зависят также от интенсивности падающей волны. В этом параграфе, основываясь на формулах для коэффициентов прохождения и отражения, мы будем исследовать кривые качания в зависимости от интенсивности падающей волны.

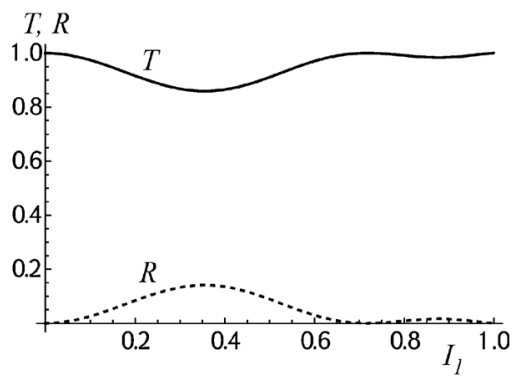

Рис.4. Кривые качания в зависимости от интенсивности для случая $\alpha=-60^{\circ}, y_{0}=-2$; сплошная кривая - кривая проходящей волны, пунктир - кривая качания дифрагированной волны.

Рассмотрим случай $\alpha=-60^{\circ}$. Будем рассматривать коэффициенты прохождения и отражения на глубинах $z=2 \Lambda_{\mathrm{L}}\left(y_{0}\right)$. Во всех случаях поглощение ничтожно и выражения для коэффициентов прохождения и отражения, приведенные в [5], можно применять. При $y_{0}=-2 \quad Q>0$ для всех значений интенсивностей [5]. Применяя соответствующие формулы [5] и беря $z=2 \Lambda_{\mathrm{L}}\left(y_{0}=-2\right)=18.2$ мкм, находим зависимость коэффициентов прохождения и отражения от интенсивности. Эти зависимости показаны на рис.4. Напомним еще раз, что в линейном случае кривые качания не зависят от интенсивности.

Отдельно приведем пример, когда $Q$ может быть как больше, так и меньше нуля. Берем $\alpha=-60^{\circ}, y_{0}=-0.5$, глубину $z=2 \Lambda_{\mathrm{L}}\left(y_{0}=-0.5\right)=36.3$ мкм. График

зависимости знака $Q$ приведен на рис.5. Видно, что начиная со значения 


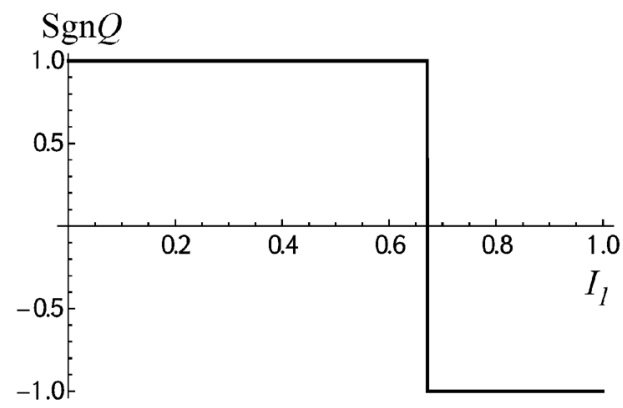

Рис.5. Зависимость знака функции $Q$ от интенсивности для $\alpha=-60^{\circ}$ и $y_{0}=-0.5$.

$I_{1}=0.67$ знак $Q$ меняется от положительного к отрицательному. Поэтому, в области до этого значения кривые качания определяются формулой соответствующей $Q>0$, а начиная с этого значения интенсивности, кривые качания определяются формулой, соответствующей $Q<0$ [5]. На рис.6 показаны кривые качания в зависимости от интенсивности во всей области изменения. Как видно, поведение кривых качаний меняется начиная со значения интенсивности $I_{1}=0.67$. В этой области энергия в основном сконцентрирована в проходящем пучке.

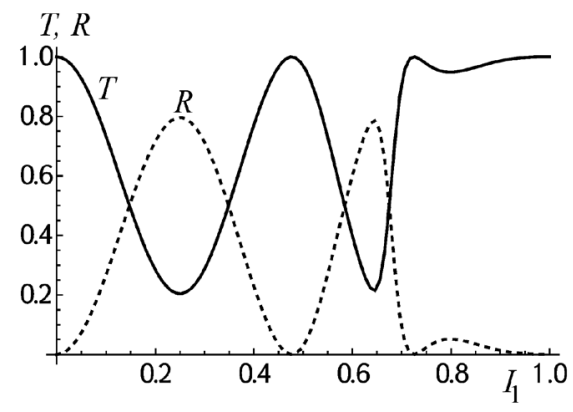

Рис.6. Кривые качания в зависимости от интенсивности для случая $\alpha=-60^{\circ}, y_{0}=-2 ;$ сплошная кривая - кривая проходящей волны, пунктир - кривая качания дифрагированной волны.

\section{4. Заключение}

Кривые качания являются одним из важных и практически применяемых характеристик динамической дифракции рентгеновских лучей. Если в области малых интенсивностей, где применима линейная теория поляризуемости, эти кривые хорошо изучены как теоретически, так и экспериментально, то же самое нельзя сказать для высокоинтенсивных пучков, для которых важны нелинейные члены в поляризуемости кристалла. В настоящее время высокоинтенсивные источники рентгеновского излучения - синхротроны и рентгеновские лазеры на свободных электронах, побудили интерес к теоретическому и эксперименталь- 
ному изучению нелинейных рентгеновских эффектов. В этой работе рассмотрены кривые качания кубически-нелинейной брэгговской асимметричной дифракции в геометрии на прохождение. Полученные ранее точные решения позволили построить кривые качания во всей области изменения независимых параметров, таких как параметр отклонения от условия Брэгга и интенсивность падающей волны. Кривые качания получены для различных углов асимметрии.

Полученные результаты могут быть применены в дальнейших теоретических и экспериментальных исследованиях нелинейной рентгеновской дифракции, а также для получения пучков с заданными параметрами или же для исследования объектов.

\section{ЛИТЕРАТУРА}

1. A. Authier. Dynamical Theory of X-ray Diffraction, Oxford: University Press, 2001.

2. З. Г. Пинскер. Рентгеновская кристаллооптика, Москва: Наука, 1982.

3. M.K. Balyan. Crystallogr. Rep., 60, 993 (2015).

4. M.K. Balyan. J. Synchrotron Rad., 22, 1410 (2015).

5. M.K. Balyan. Physica scripta, 96, 125006 (21) (2021).

6. M.K. Balyan. J. Contemp. Phys., 51, 391 (2016).

7. M.K. Balyan. J. Synchrotron Rad., 23, 919 (2016).

8. I. Freund, B. F. Levine. Phys. Rev. Lett., 23, 854 (1969).

9. P. Eisenberger, S. L. McCall. Phys. Rev. Lett., 26, 684 (1971).

10. B. Adams, P. Fernandez, W-K. Lee, G. Materlik, D. Mills, D. Novikov. J. Synchrotron Rad., 7, 81 (2000).

11. Y.T. Yoda, T. Suzuki, X-W. Zhang, K. Hirano, S. Kikuta. J. Synchrotron Rad., 5, 980 (1998).

12. S. Shwartz, R.N. Coffe, J.M. Feldkamp, Y. Feng, J.B. Hastings, G.Y. Yin, S.E. Harris. Phys. Rev. Lett., 109, 013602 (2012).

13. A. Schori, C. Bömer, D. Borodin, S. Collins, B. Detlefs, M. Morett, S. Yudovich, S. Shwartz. Phys. Rev. Lett., 119, 253902 (2017).

14. H. Danino, I. Freund. Phys. Rev. Lett., 46, 1127 (1981).

15. K. Tamasaku, T. Ishikawa. Phys. Rev. Lett., 98, 244801 (2007).

16. K. Tamasaku, T. Ishikawa. Acta Cryst., A63, 437 (2007).

17. D. Borodin, S. Levy, S. Shwartz. Appl. Phys. Lett., 110, 131101 (2017).

18. B. Barbiellini, Y. Joly, K. Tamasaku. Phys. Rev. B, 92, 155119 (2015).

19. D. Borodin, A. Schori, J.-P. Rueff, J.M. Ablett, S. Shwartz. Phys. Rev. Lett., 122, 023902 (2019).

20. A. Nazarkin, S. Podorov, I. Uschmann, E. Förster, R. Sauerbrei. Phys. Rev. A, 67, 041804 (2003).

21. S. Shwartz, M. Fuchs, J.B. Hastings, N. Inubushi, T. Ishikawa, T. Katayama, D.A. Reis, T. Sato, K. Tono, M. Yabashi, S. Yudovich, S.E. Harris. Phys. Rev. Lett., 
112, 163901 (2014).

22. M. Fuchs, M. Trigo, J. Chen, Sh. Ghimire, Sh. Shwartz, M. Kozina, M. Jiang, T. Henighan, C. Bray, G. Ndabashimiye, Ph. Bucksbaum, Y. Feng, S. Herrmann, G. Carini, J. Pines, Ph. Hart, Ch. Kenney, S. Guillet, S. Boutet, G.J. Williams, M. Messerschmidt, M.M. Seibert, S. Moeller, J.B. Hastings, D. Reis. Nature Physics, 11, 964 (2015).

23. D. Krebs, D. Reis, R. Santra. Phys. Rev. A, 99, 022120 (2019).

24. C. Conti, A. Fratallocchi, G. Ruocco, F. Sette. Opt. Express, 16, 8324 (2008).

25. S. Tanaka, S. Mukamel. Phys. Rev. Lett., 89, 043001 (2002).

26. K. Tamasaku, E. Shigemasa, Y. Inubushi, T. Katayama, K. Sawada, H. Yumoto, H. Ohashi, H. Mimura, M. Yabashi, K. Yamauchi, T. Ishikawa. Nature Photon Lett., 8, 313 (2014).

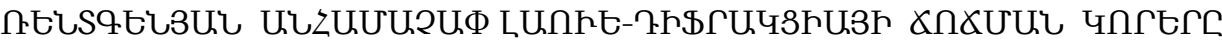

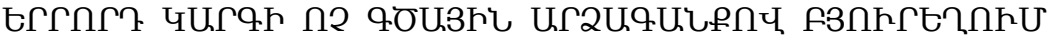

\section{U.Ч. FULBU乙}

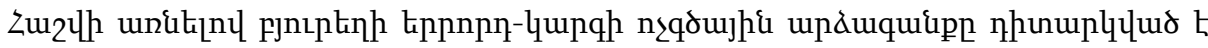

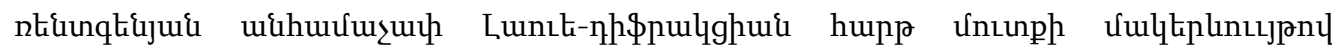

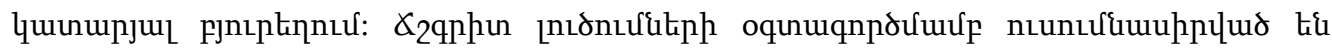

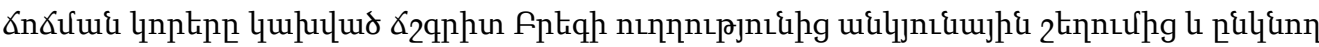

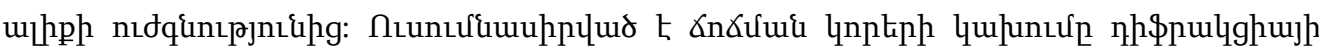

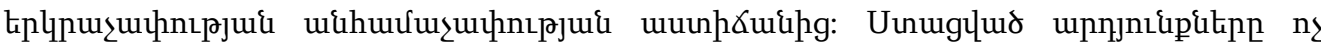

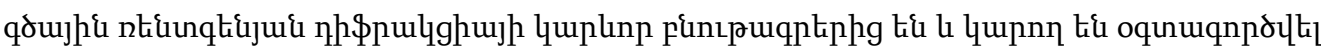

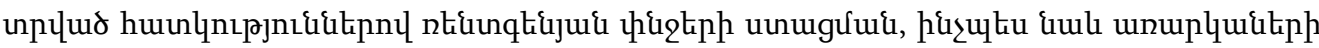
nıunıर्uquuhphưu hưưp:

\section{ROCKING CURVES OF X-RAY ASYMMETRICAL LAUE DIFFRACTION IN A CRYSTAL WITH THE THIRD-ORDER NONLINEAR RESPONSE}

\section{M.K. BALYAN}

Taking into account the third-order nonlinear response of a crystal, the X-ray Laue case asymmetrical diffraction in a perfect crystal with a plane entrance surface is considered. Using the exact solutions the rocking curve dependences on the angular departure from the exact Bragg direction and on the intensity of the incident wave are investigated. The dependence of rocking curves on asymmetry degree of diffraction geometry is investigated. The obtained results can be used for preparation of X-ray beams with the given parameters and for object investigations as well. 